\title{
Recognition and Characterization of Stage-specific Oocyst/Sporozoite Antigens of Toxoplasma gondii by Human Antisera
}

\author{
Lloyd H. Kasper and Patricia L. Ware \\ Department of Medicine (Neurology), Dartmouth Medical School, Hanover, New Hampshire 03756
}

\begin{abstract}
Human infection with Toxoplasma gondii is presumed due to the ingestion of either tissue cysts containing bradyzoites or oocyst/sporozoites that are excreted in the feces of infected cats. The incidence of human infection in the general population by either of these routes is unknown. We have previously described unique stage-specific oocyst/sporozoite antigens identified by murine hybridoma monoclonal antibodies. We obtained acute and convalescent antitoxoplasma antisera from patients in an epidemiologically well-documented outbreak of oocyst-transmitted infection associated with the ingestion of contaminated water. An enzyme-linked immunosorbent assay comparing equal numbers of tachyzoites (invasive stage) and oocyst/sporozoite (excreted stage) indicated that these antisera recognized antigens from both life forms. Absorption of pooled antisera with purified oocyst/sporozoites reduced both the antioocyst immunoglobulin $\mathbf{G}$ (IgG) and immunoglobulin $\mathbf{M}$ (IgM) titer but had only minimal effect on the antitachyzoite titer. Absorption of the antisera with tachyzoites reduced the IgG and IgM antioocyst and antitachyzoite titer. A sodium dodecyl sulfate-polyacrylamide gel analysis of radioiodinated oocyst/sporozoites shows that the principal stage-specific surface proteins of the oocyst/sporozoite have approximate $M_{\mathrm{r}}$ of 67,000 and 25,000 . Periodic acid and silver stain of purified oocyst/sporozoite identified bands of similar molecular weight not present in the tachyzoite preparation. Western blot analysis of purified parasites assayed with human antioocyst antisera identified specific oocyst/sporozoite antigens not present on the tachyzoites. At least two major stage-specific oocyst/ sporozoite antigens of approximate $M_{\mathrm{r}}$ of 67,000 and 190,000 were identified by the infected patients' antisera and not by the normal controls. Reaction to these oocyst/sporozoite antigens was seen primarily in the IgM fraction of the acute phase and the IgG fraction of convalescent phase antisera. Neither absorption of the antisera with tachyzoites nor periodate treatment of the oocyst/sporozoites reduced the antibody recognition of these stage-specific antigens. These data suggest that individuals infected by a presumed oocyst-transmitted route develop antibodies against unique stage-specific oocyst/ sporozoite antigens.
\end{abstract}

\section{Introduction}

Human infection with Toxoplasma gondii is presumed to be due to ingestion of either oocysts or tissue cysts containing

Received for publication 14 June 1984 and in revised form 14 January 1985.

J. Clin. Invest.

(c) The American Society for Clinical Investigation, Inc.

0021-9738/85/05/1570/08 \$1.00

Volume 75, May 1985, 1570-1577 bradyzoites. Although the asexual stage of $T$. gondii was first described 75 years ago, it was not until a decade ago that the sexual cycle in cats was understood (1-4). This enteroepithelial cycle usually begins with the ingestion of the tissue cysts and culminates after several intermediate stages with the shedding of infectious oocysts in the feces.

Recently, we reported on the identification of stage-specific oocyst/sporozoite antigens of $T$. gondii by monoclonal antibodies (5). Several of these monoclonal antibodies failed to react with $T$. gondii tachyzoite surface membrane antigens. Radioiodination of the oocyst/sporozoite surface membrane, followed by one- and two-dimensional electrophoresis showed that $T$. gondii oocyst/sporozoites had several membrane proteins not present on the tachyzoite. The two major protein antigens to which monoclonal antibody was directed had approximate $M_{\mathrm{r}}$ of 25,000 and 67,000 (designated SP25 and sP67). A convalescent human antitoxoplasma serum failed to recognize oocyst/sporozoite antigens by both immunoprecipitation and reaction with nitrocellulose transfer blots.

We and others have reported on the various tachyzoite surface membrane and cytoplasmic antigens identified by acute and convalescent human antitoxoplasma antisera (611). Oocyst transmission to humans has been described epidemiologically by several authors (11-14). However, immunologic documentation of oocyst transmission has not been reported. We obtained acute and convalescent human antisera from eight infected and two control patients in an epidemiologically well-documented outbreak of oocyst-transmitted infection associated with the ingestion of contaminated water (15). These sera were assayed for stage-specific antioocyst/ sporozoite antibody. We now report further biochemical and immunologic analysis of the oocyst/sporozoite antigens of $T$. gondii as well as the recognition of these stage-specific antigens by human antioocyst antisera.

\section{Methods}

Parasites and host cells. The methods for growth of $T$. gondii in cultured human fibroblasts (HF) ${ }^{1}$ have been described (16). The three types of tachyzoites used in these experiments were either a cloned line of the RH strain, or freshly isolated C or P strain. The RH strain tachyzoite has been maintained in our laboratory by continuous passage in HF cells. Both oocyst/sporozoites and bradyzoites become rapidly dividing tachyzoites when cultured in HF cells. C strain tachyzoites were obtained by mechanical fracture of oocysts followed by subculture of the sporozoites in HF. All experiments involving C strain tachyzoites were carried out between the 1st and 40th passage of the parasites in culture. P strain tachyzoites were obtained from the M-47 line of T. gondii (kindly supplied by Dr. M. Lunde, National Institutes of Health). These parasites produce high numbers of brain tissue cysts in mice. Bradyzoites were obtained by homogenization of

1. Abbreviations used in this paper: ELISA, enzyme-linked immunosorbent assay; HF, human fibroblasts; PAGE, polyacrylamide gel electrophoresis. 
cyst-infected brains, followed by forced passage through a 30-gauge needle. Parasites were then subcultured in HF cells. The resulting $P$ strain tachyzoites were used between the 2 nd and 15 th passage.

Tachyzoites were obtained from infected monolayers by forced extrusion through a 27-gauge needle. Residual host cell debris was removed by resuspending the centrifuged parasite sediment in PBS, pH 7.4, that contained $0.2 \mathrm{~g} / \mathrm{ml}$ phytohemagglutinin P (Difco Laboratories Inc., Detroit, $\mathrm{MI})(17)$. After agitation for $30 \mathrm{~min}$ at $37^{\circ} \mathrm{C}$ the parasites were passed through a loosely packed nylon wool column and centrifuged. This procedure resulted in an $80-90 \%$ recovery of the RH strain and a $25-30 \%$ recovery of $\mathrm{C}$ and $\mathrm{P}$ strain tachyzoites.

Oocysts were obtained in a manner similar to that previously described (18). Briefly, mice were infected with $C$ strain tachyzoites and treated with $0.25 \mathrm{mg} / \mathrm{ml}$ sulfadiazine in their drinking water when they appeared ill. After 6 wk the mice were killed and an estimated 1,000 cysts were fed to kittens. Feces were collected daily and purified by flotation in a sucrose gradient. The oocysts were allowed to sporulate at room temperature for several days in $0.5 \mathrm{~N}$ sulfuric acid and stored at $4^{\circ} \mathrm{C}$. To prepare crude oocyst/sporozoite lysates, oocysts were washed several times in Hanks' buffered saline solution and freeze thawed five times in an ethanol/dry ice bath. The suspension was then sonicated eight times $(18,000 \mathrm{~Hz})$ at 15 -s intervals over $4 \mathrm{~min}$ at $4^{\circ} \mathrm{C}$ and stored at $-20^{\circ} \mathrm{C}$.

Assay of antitoxoplasma antibody. Human antitoxoplasma antisera was provided by a generous gift from Dr. E. Takafuji (Division of Preventive Medicine, Walter Reed Army Institute of Research, Washington, DC). The antisera were from a well-documented epidemiologic study of oocyst-transmitted toxoplasmosis associated with the ingestion of contaminated water (15). Control antisera were obtained from the same patient group. These control individuals were part of the same platoon of soldiers but did not demonstrate clinical signs or symptoms of infection and had a nominally elevated antibody titer as determined by enzyme-linked immunosorbent assay (ELISA) (immunoglobulin $G$ [IgG] < 64, immunoglobulin $M$ [IgM] < 4). Other control sera were obtained from laboratory personnel with IgG antitoxoplasma tite $<64$ as determined by an ELISA. We measured antitoxoplasma antibody with an ELISA that has been described for both tachyzoite and oocyst/sporozoite antigens $(5,6,17)$. Equal numbers of purified oocyst/sporozoites and tachyzoites were deposited in 96-well plates (Linbro). After blocking with 5\% fetal bovine serum, human antisera was incubated for $2 \mathrm{~h}$ at $37^{\circ} \mathrm{C}$. Antibody binding was identified with peroxidase labeled goat antihuman IgG and IgM (Cappel Laboratories, Inc., Cochranville, PA).

Polyacrylamide gel electrophoresis (PAGE) and radioiodination of proteins. Parasite samples were dissolved in sample buffer that contained 2\% SDS and 5\% 2-mercaptoethanol. After boiling for $3 \mathrm{~min}$, samples were electrophoresed in a $0.1 \%$ SDS discontinuous $10 \%$ polyacrylamide gel. Gels were run on a Mini Slab apparatus (Idea Scientific, Corvalis, OR) to reduce the amount of sample needed. The molecular weight standards include carbonic anhydrase $(29,000)$, egg albumin $(45,000)$, bovine albumin $(66,000)$, phosphorylase B $(97,000)$, B galactosidase $(116,000)$, and myosin $(205,000)$ (Sigma Chemical Co., St. Louis, MO).

Surface proteins of $T$. gondii were radioiodinated by previously reported IODOGEN technique (6). After iodination the parasites were dissolved in 0.5\% NP-40 nonionic detergent (Particle Data, Inc., Elmhurst, IL) and stored at $-20^{\circ} \mathrm{C}$.

Silver staining. After electrophoresis the polyacrylamide gels were soaked in $50 \%$ methanol overnight. Silver stain was prepared fresh each day according to the method of Wray et al. (19). Briefly, gels were stained in a solution of $0.8 \%$ silver nitrate in $0.021 \mathrm{M}$ sodium hydroxide and $0.2 \mathrm{M}$ ammonium hydroxide. They were washed for at least $5 \mathrm{~min}$ in distilled water and developed in a solution of $0.005 \%$ citric acid and $0.019 \%$ formaldehyde. Gels were rinsed briefly with distilled water, soaked overnight in $50 \%$ methanol, and brought back to original size by soaking in $20 \%$ methanol.

Periodic acid silver stain. We used a modification of the method of Dubray and Bezard (20). After electrophoresis gels were soaked in
$25 \%$ isopropyl alcohol and $10 \%$ acetic acid overnight. After a rinse in distilled water the gels were soaked in $0.02 \%$ aqueous periodic acid at $4^{\circ} \mathrm{C}$ for $1 \mathrm{~h}$. They were then washed with several rinses of distilled water for $3 \mathrm{~h}$, stained $15 \mathrm{~min}$ with the standard silver stain, and developed as described.

Nitrocellulose blot transfer electrophoresis. A modification of the technique first described by Towbin et al. (21) was used. After SDSPAGE, gels were applied to a sheet of $0.2-\mu \mathrm{m}$ pore nitrocellulose paper (Schleicher \& Schuell, Inc., Keene, NH) and electrophoresed for $3 \mathrm{~h}$ with a 6-A battery charger. After electrophoresis the remaining protein binding sites on the nitrocellulose paper were blocked with $3 \%$ bovine serum albumin in PBS, pH 7.4, overnight at $4^{\circ} \mathrm{C}$. The nitrocellulose was washed several times in $0.05 \%$ Tween 20 , PBS. Strips were cut from the nitrocellulose paper and incubated with dilute antisera (either 1:20 or 1:50) in blocking buffer for $2 \mathrm{~h}$ at room temperature. The strips were washed and incubated with peroxidase-labeled goat antihuman IgG or IgM (1:2000 and 1:250; Cappel Laboratories) for $1 \mathrm{~h}$. The strips were washed and developed in a solution of $0.02 \%$ diaminobenzadine, $0.1 \%$ hydrogen peroxide, $0.01 \mathrm{M}$ PBS. The reaction was stopped with an overnight saline wash and dried nitrocellulose strips were stored protected from light until photographed.

\section{Results}

The human antitoxoplasma antisera were examined for the presence of antibody to oocyst/sporozoites and tachyzoites. An ELISA comparing equal numbers of purified oocyst and RH strain tachyzoites was done for both acute and convalescent antisera. We have shown previously that both tachyzoites and sporozoites bind to a solid matrix for immunofluorescence studies (5). To demonstrate the ability of the two parasite forms to bind to a solid substrate, equal numbers of sonicated radioiodinated oocyst/sporozoites and tachyzoites were deposited in microtiter plates, blocked with $5 \%$ FBS, washed, and counted. By this method, it was determined that oocyst/ sporozoites adhere equally well as tachyzoites to microtiter plates. For the ELISA test, $2.5 \times 10^{4}$ sporulated oocysts per well provided the antigen source (eight infectious sporozoites per oocyst). RH strain tachyzoites at a concentration of 2.0 $\times 10^{5}$ parasites per well were prepared in a similar manner and used in a parallel assay. Using peroxidase-labeled antihuman IgG and IgM, we were able to demonstrate antitachyzoite and antioocyst activity. As shown in Table I there was an increase in antibody titer to $T$. gondii between the acute and convalescent antisera, in particular the IgG fraction. Generally, antibody titer to tachyzoites was greater than antibody to oocysts. The acute phase antitachyzoite IgG tier was slightly greater than the antioocyst titer. This difference in IgG antibody titer between the tachyzoites and oocysts was less apparent in the convalescent antisera. The IgM titer of acute and convalescent antisera to oocysts and tachyzoites was less than the IgG titer. The IgM antitachyzoite titer is similar to the antioocyst titer in both the acute- and convalescent-phase antisera.

The antiserum from several patients with a high antioocyst/ sporozoite titer was selected for further study. A dilution of the pooled antisera (1:100) was absorbed with equal numbers of either purified oocyst or RH strain tachyzoites. Absorption was done by incubating $0.5 \mathrm{ml}$ of dilute antisera with 2.5 $\times 10^{8}$ parasites at $37^{\circ} \mathrm{C}$ for $2 \mathrm{~h}$. After absorption, a parallel ELISA was done comparing identical volumes of lysate that contained equal numbers of oocyst/sporozoites and tachyzoites. Both acute and convalescent antisera were assayed. Table II shows that the antioocyst titer of the pooled acute-phase 
Table I. Elisa Titer of Acute and Convalescent Human Antitoxoplasma Antisera to Purified Tachyzoites and Oocyst/Sporozoites

\begin{tabular}{|c|c|c|c|c|c|c|c|c|}
\hline \multirow[b]{3}{*}{ Patient $n=8$} & \multicolumn{4}{|c|}{ Acute antisera } & \multicolumn{4}{|c|}{ Convalescent antisera } \\
\hline & \multicolumn{2}{|l|}{ IgG } & \multicolumn{2}{|l|}{ IgM } & \multicolumn{2}{|l|}{ IgG } & \multicolumn{2}{|l|}{ IgM } \\
\hline & Oocyst & Tachyzoite & Oocyst & Tachyzoite & Oocyst & Tachyzoite & Oocyst & Tachyzoite \\
\hline 71 & 2,048 & 4,096 & 1,024 & 1,024 & 16,384 & 16,384 & 512 & 512 \\
\hline 87 & 512 & 4,096 & 256 & 256 & 16,384 & 16,384 & 2,048 & 1,024 \\
\hline 74 & 256 & 1,024 & 1,024 & 1,024 & 8,192 & 8,192 & 2,048 & 1,024 \\
\hline 93 & 1,024 & 2,048 & 512 & 1,024 & 8,192 & 16,384 & 512 & 256 \\
\hline 82 & 1,024 & 4,096 & 512 & 1,024 & 8,192 & 16,384 & 2,048 & 4,096 \\
\hline 68 & 1,024 & 8,192 & - & - & 4,096 & 8,192 & 512 & 512 \\
\hline 64 & 512 & 2,048 & 64 & 256 & 16,384 & 16,384 & 512 & 1,024 \\
\hline 109 & 1,024 & 512 & - & - & 4,096 & 1,024 & 512 & 512 \\
\hline Control $\times 2$ & 32 & 64 & 16 & 16 & 32 & 32 & 4 & 16 \\
\hline
\end{tabular}

antisera was reduced when absorbed with oocyst/sporozoites. This severalfold reduction was seen with both the IgG and IgM antioocyst fractions. Absorption of the antisera with oocysts had no effect on the IgG antitachyzoite titer. The IgM antitachyzoite titer was reduced in half by absorption with oocyst/sporozoites. Absorption of the antisera with tachyzoites uniformly reduced the antitachyzoite and antioocyst IgG titer. There was a reduction in the antitachyzoite IgM titer between oocyst and tachyzoite absorbed antisera. Absorption had no effect on the low titer control antisera. Similar findings were seen with the convalescent antisera (data not shown).

Although not specific, the absorption study suggested that human antitoxoplasma antisera identified oocyst antigens. A more detailed analysis of the oocyst/sporozoite antigens recognized by this antisera was necessary. Our previous study had shown that radioiodinated oocyst/sporozoites differed from both RH and C strain tachyzoites. Fig. 1 illustrates a onedimensional SDS-PAGE of equal numbers of surface membrane-radioiodinated tachyzoites and oocyst/sporozoites. As noted previously there is the striking presence of two major iodinated oocyst/sporozoite proteins of approximate $M_{\mathrm{r}} 67,000$ and 25,000 not present in the tachyzoite preparation. On prolonged radiographic exposure ( $3 \mathrm{wk})$ at least three other unique proteins not previously reported become apparent. This includes two high $M_{\mathrm{r}}$ protein bands $(>100,000)$ and one

Table II. ELISA Titer of Parasite-absorbed Human

Antitoxoplasma Antisera* to Purified

Tachyzoites and Oocyst/Sporozoites

\begin{tabular}{|c|c|c|c|c|}
\hline \multirow[b]{2}{*}{ Antisera } & \multicolumn{2}{|l|}{ IgG } & \multicolumn{2}{|l|}{ IgM } \\
\hline & Tachyzoite & Oocyst & Tachyzoite & Oocyst \\
\hline \multicolumn{5}{|l|}{$\begin{array}{l}\text { Antitoxoplasma positive } \\
\quad(n=3)\end{array}$} \\
\hline Without absorption & 4,096 & 2,048 & 1,024 & 1,024 \\
\hline Oocyst absorbed & 4,096 & 64 & 512 & 128 \\
\hline Tachyzoite absorbed & 64 & 64 & 64 & 128 \\
\hline \multicolumn{5}{|l|}{$\begin{array}{l}\text { Antitoxoplasma negative } \\
\quad(n=3)\end{array}$} \\
\hline Without absorption & 64 & 64 & 16 & 16 \\
\hline Tachyzoite absorbed & 32 & 32 & 16 & 8 \\
\hline
\end{tabular}

* Pooled acute-phase antisera. other band of approximate $M_{\mathrm{r}} 55,000$. There are also unique tachyzoite proteins $(14,000,22,000,30,000,43,000$, and 97,000 $M_{\mathrm{r}}$ ) that have been described previously. Several proteins appear to be shared in common with tachyzoites and oocyst/ sporozoites. Both preparations exhibit a dark band below the $14,000 M_{\mathrm{r}}$ marker that most likely represents iodinated proteins and lipids. Separation of these low molecular weight oocyst/ sporozoite antigens has not yet been achieved.

Identification of radioiodinated surface membrane antigens is limited to those proteins which can be labeled with this technique. Our previous report examined only those stage specific antigens that could be labeled by radioiodination. We therefore used the highly sensitive silver and periodic acidsilver stain for protein and carbohydrates to detect any further proteins, glycoproteins, or polysaccharides that may biochemically distinguish the oocyst/sporozoite from the tachyzoites. First, it was necessary to document any tachyzoite strain specific antigens that would make interpretation of stage specificity unclear. To do this, we compared the laboratory RH wild type with freshly isolated $C$ and $P$ strain tachyzoites. Equal numbers of purified tachyzoites $\left(1 \times 10^{6}\right)$ were solubilized in PBS $0.05 \%$ nonionic detergent and run on $10 \%$ SDS-PAGE. Protein and carbohydrate bands were identified as described in Methods. Fig. 2 shows a comparison of the silver- and periodic acid-stained bands. The crude tachyzoite extracts show the expected extensive array of silver-stained protein bands. Numerous bands common to all three tachyzoite strains

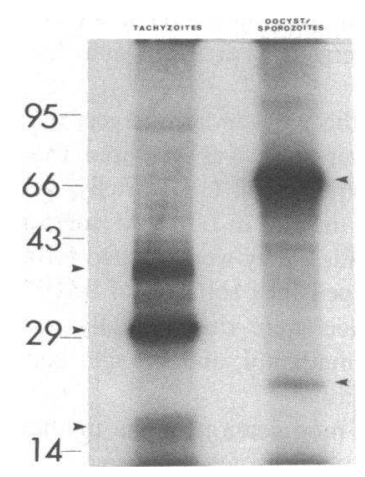

Figure 1. Autoradiographic comparison of SDS-polyacrylamide gels of radioiodinated $T$. gondii. Equal numbers $\left(5 \times 10^{7}\right)$ of tachyzoites and oocyst/sporozoites were radiolabeled in parallel by the Iodogen method and solubilized in an equal volume of $0.5 \%$ NP-40 nonionic detergent and phosphate-buffered saline. Equal volumes of radioiodinated parasites (1.9 $\times 10^{5} \mathrm{cpm} / 10 \mu \mathrm{l}$ for tachyzoites and $2.4 \times 10^{5} \mathrm{cpm} / 10 \mu \mathrm{l}$ for oocyst/sporozoites) were run on $10 \%$ polyacrylamide gels in the presence of SDS. Gels were dried and autoradiographed for $9 \mathrm{~d}$. 


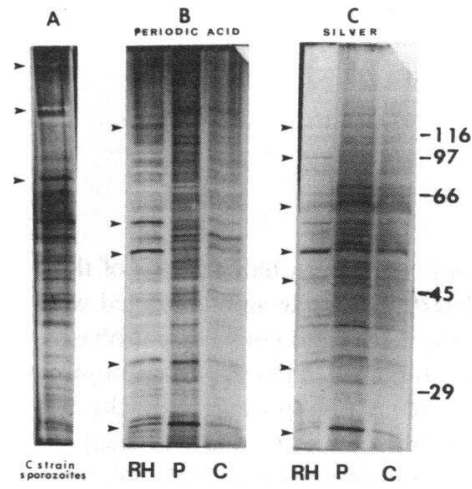

hydrates of oocyst/sporozoite preparation. (B) Periodic acid stain of RH laboratory strain tachyzoites, $P$ strain tachyzoites recovered from mouse brain tissue cysts containing bradyzoites and $C$ strain tachyzoites recovered from oocyst/sporozoites. $(C)$ Silver stain for protein of the same preparation of RH strain, P strain, and C strain tachyzoites.

can be seen. These include major bands at $14,000,30,000$, $43,000,48,000,64,000$, and $97,000 M_{\mathrm{r}}$ as well as two bands $>100,000$. There are also numerous minor proteins that are shared by these three strains. There do appear to be several minor staining protein bands that are present primarily in the $C$ and $P$ strain and not the RH strain preparation. These bands could represent minor tachyzoite strain-specific proteins. Similarly, the periodic acid stain revealed common bands among the three tachyzoite strains. These included bands at $M_{\mathrm{r}} 14,000,30,000,52,000,64,000$, and $>100,000$. Of note, with periodic acid staining there were numerous clear bands that failed to stain in all three tachyzoite preparations. Tachyzoite strain variation appeared to be more prominent when the periodic acid-positive bands of the three tachyzoite preparations were evaluated. Overall, there was a greater number of periodic acid-sensitive carbohydrate bands in comparison to silver-sensitive protein bands. In general, the major proteinand carbohydrate-containing determinants are shared by the three different tachyzoite strains.

Although these results show that the three tachyzoite strains investigated share similar major proteins and carbohydrates, we felt that for the analysis of sporozoite-specific antibody in human sera, parasites from complete strains that form tachyzoites, bradyzoites, and sporozoites should be compared. Both the $\mathbf{P}$ and $\mathbf{C}$ strains are adequate for this purpose. Fig. 3 shows a Western blot analysis of our three different tachyzoite strains ( $\mathrm{P}, \mathrm{C}$ and $\mathrm{RH}$ ) reacted with pooled acute and convalescent-phase antitoxoplasma antisera from the study group. Tachyzoites were purified from HF cells as described in Methods. Equal numbers of tachyzoites $\left(2 \times 10^{6} /\right.$ lane $)$ were run on a single $10 \%$ polyacrylamide gel in the presence of SDS and electrophoretically transferred to nitrocellulose paper. Lanes were incubated with either acute or convalescent-phase antisera. Antibody binding was determined with either peroxidase labeled goat anti-human IgG or IgM. As seen in Fig. 3 there is homogeneity among the major tachyzoite antigens in these three different strains. Among the more active bands are antigens at approximate $M_{\mathrm{r}} 22,000,30,000,52,000$, and 72,000 . There are 7-10 minor antigens that are commonly shared among the three strains. In addition, there are various minor strain-specific antigens that appear to be unique to each of the individual strains. Thus, in general, the major tachyzoite antigens identified by the acute- and convalescent-phase antitoxoplasma antisera are shared in common among the three tachyzoite strains investigated.

We next analyzed the protein and carbohydrate content of the oocyst/sporozoite and tachyzoite. This was necessary to distinguish any minor variation in antigenic determinants not recognized by surface radioiodination. Because all three tachyzoite strains appeared to contain common major antigens, the RH strain tachyzoite was chosen for further study. Fig. 4 is a 10\% SDS-PAGE that compares equal numbers of $\mathrm{RH}$ strain tachyzoites and $\mathrm{C}$ strain oocyst/sporozoites. Both parasites (lanes $A$ and $B$ ) show numerous bands of similar molecular weight when stained for protein with silver. These include several of the shared tachyzoite antigens that were previously described. There are 10-12 protein bands present in the oocyst preparation not seen in the tachyzoites. Included in this group of stage-specific proteins are several weakly silver staining bands of approximate $M_{\mathrm{r}} 25,000,40,000$, and 67,000. Interestingly, bands of similar molecular weight were the most intensely labeled proteins by surface radioiodination.

A periodic acid carbohydrate stain for analysis of polysaccharide, glycoprotein, and glycolipid determinants was done. Lanes $C$ and $D$ compare tachyzoites with oocysts. Again there are many corresponding bands between these two stages. However, there are 9-10 bands in the oocyst/sporozoite not present in the tachyzoite preparation. Among these unique determinants are strongly stained carbohydrate-containing bands at $M_{\mathrm{r}} 25,000,67,000$ and a nonstaining doublet in the 190,000 range. Numerous bands are present in the periodic acid-stained oocyst and tachyzoite preparation that are not clearly identified by silver staining. On the other hand, silver staining of the oocyst and tachyzoite revealed protein bands not detected by the periodic acid staining procedure.

The most sensitive method for identifying unique protein antigens that may differ between the tachyzoite and oocyst/ sporozoite is by Western blot analysis. We chose for comparison the RH strain tachyzoite because our previous results (Figs. 2 and 3) indicated that all three tachyzoite strains exhibited biochemical and immunologic similarity. Fig. 5 shows the results of equal numbers $\left(2 \times 10^{4}\right)$ of purified tachyzoites (lanes $a$ and $c$ ) and oocyst/sporozoites (lanes $b$ and $d$ ) run on $10 \%$ SDS-PAGE and transferred to nitrocellulose. After incubation with acute antisera, the IgG and IgM binding antigens were identified. There are at least five stage specific oocyst/sporozoite antigens recognized by the acute IgG fraction that are not present in the tachyzoite preparation. These antigen bands range in $M_{\mathrm{r}}$ from 25,000 to 190,000 . The most prominent of these bands occur at $M_{\mathrm{r}} 25,000,67,000$, and 190,000. There are also at least three stage-specific tachyzoite antigens not present on the oocyst/sporozoite $\left(M_{\mathrm{r}} 30,000,62,000\right.$, and $>100,000)$. Numerous commonly shared oocyst-tachyzoite antigens can be identified by this antisera. This includes major bands at $M_{\mathrm{r}} 22,000,44,000,66,000$, and 97,000 . When the antisera from each of the eight patients were individually assayed, a similar pattern of stage-specific antigen recognition was observed. Intensity of antibody binding by the different antigens was the major variation among this group of patients. Four control patients (two from the cohort and two laboratory personnel with negative ELISA titers) failed to identify any of the major tachyzoite or oocyst/sporozoite antigens. 

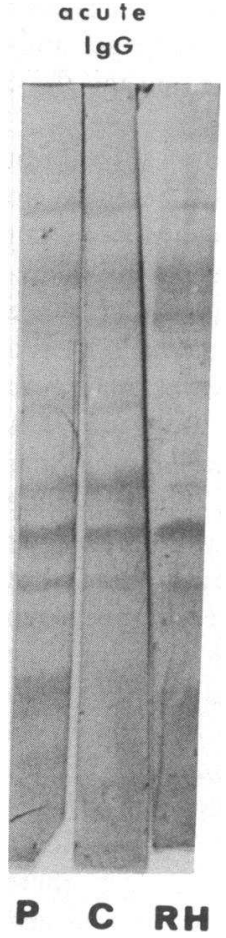

convalescen $\dagger$

l g G

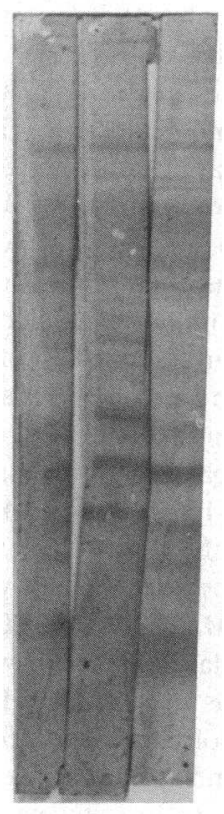

P C RH

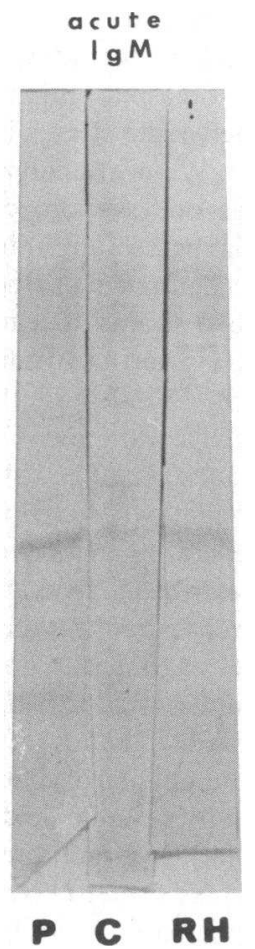

P C RH convalescent I g $M$

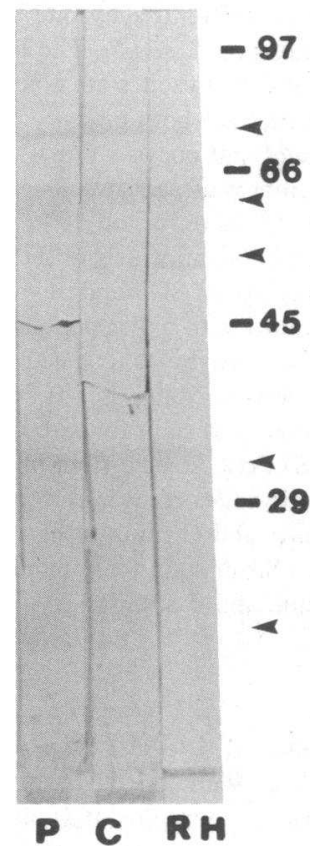

Figure 3. Western blot analysis of three different tachyzoite strains reacted with pooled acute- and convalescent-phase antitoxoplasma antisera. Equal numbers $\left(2 \times 10^{6}\right)$ of column purified tachyzoites from the P, C, and RH tachyzoite strain were run on $10 \%$ polyacrylamide gels in the presence of SDS and electrophoretically transferred to nitrocellulose paper. Transferred nitrocellulose strips were incubated with either pooled acute- or convalescent-phase antisera. Antibody binding was determined by peroxidase labeled goat antihuman IgG and IgM antibody. (A) Acute-phase antisera, IgG fraction. (B) Convalescent-phase antisera, IgG fraction. $(C)$ Acute-phase antisera, IgM fraction. (D) Convalescent-phase antisera, IgM fraction.
The acute IgM antisera fraction also expresses sporozoite/ oocyst stage specificity. Unique sporozoite antigens can be seen from $\sim 38,000$ to $190,000 M_{\mathrm{r}}$. Three noteworthy sporozoite antigens occur at $M_{\mathrm{r}} 38,000,67,000$, and 190,000 . There are numerous minor tachyzoite antigens not present in the sporozoite population. Several of the sporozoite bands $(67,000$ and $190,000 M_{\mathrm{r}}$, in particular) are recognized by both the IgG and IgM acute antisera fractions. The broad $M_{\mathrm{r}} 67,000$ band seen with the IgM fraction correlates with a band of similar molecular weight identified by surface radioiodination and by two of our stage-specific monoclonal antibodies (5). There are fewer commonly shared oocyst-tachyzoite antigens identified by the IgM than by the IgG fraction.

We then assayed convalescent antisera for antibodies against sporozoite and tachyzoite antigens. Fig. 6 shows a nitrocellulose transfer using equal numbers of tachyzoites and oocyst/spo-

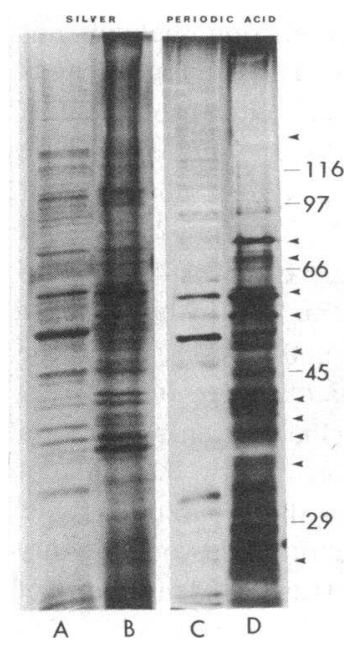

Figure 4. A comparison of equal numbers $\left(1 \times 10^{6}\right) \mathrm{RH}$ strain tachyzoites (lanes $A$ and $C$ ) and oocyst/ sporozoites (lanes $B$ and $D$ ) run on $10 \%$ polyacrylamide gels in the presence of SDS and stained by either silver or periodic acid-silver. Lanes $A$ and $B$ are stained for protein with silver. Lanes $C$ and $D$ are stained for carbohydrates with periodic acidsilver.

rozoites. As with the acute antisera, there were many antigenic bands common to both parasite stages. However, there were also several parasite antigens recognized by convalescent antisera that were unique to either the oocyst (lane $a$ ) or the tachyzoite (lane $b$ ). Stage-specific oocyst/sporozoite bands at 25,000, 67,000 , and $190,000 M_{\mathrm{r}}$ are the most prominent. Comparison of the acute- and convalescent-phase antisera indicates that the IgG titer against the 67,000 and $190,000-M_{\mathrm{r}}$ bands increases with convalescence. The convalescent IgM antisera (Fig. 7) shows an interesting reduction in affinity against several of the sporozoite antigens. The 67,000 and $190,000-M_{\mathrm{r}}$ bands although present are not as prominent as in the acute-phase IgM antisera. There is an additional low molecular weight band $(<14,000)$ not seen in the tachyzoites. Several minor antigens also distinguish the oocyst/sporozoite from tachyzoite.

The ELISA had shown that absorption of antisera with either oocysts or tachyzoites reduced the antibody titer to a value above the normal control antisera. Another absorption assay was done to investigate which antibodies were oocyst

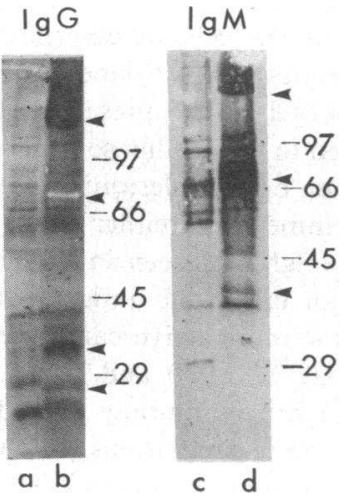

Figure 5. Nitrocellulose blot transfer of RH strain tachyzoites and oocyst/ sporozoites identified with acute-phase human antitoxoplasma antisera. Equal numbers $\left(2 \times 10^{6}\right)$ of parasites were run on $10 \%$ polyacrylamide gels in the presence of SDS and electrophoretically transferred to nitrocellulose paper. Strips were incubated with acute-phase antitoxoplasma antisera. Antibody binding was determined with peroxidase-labeled goat antihuman IgG and IgM. (Lanes $a$ and $c$ ) RH strain tachyzoites; (lanes $b$ and $d$ ) oocyst/sporozoites. 


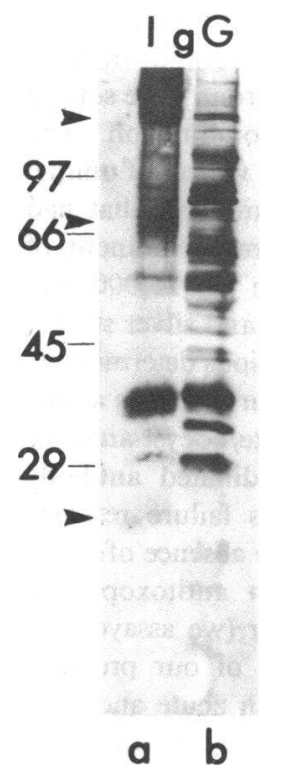

Figure 6. Nitrocellulose blot transfer of equal numbers $\left(2 \times 10^{6}\right)$ of $(a)$ oocyst/sporozoites and $(b)$ RH strain tachyzoites identified by the IgG fraction of convalescent-phase human antitoxoplasma antisera.

stage specific. Acute-phase antisera was pooled and diluted 1:100. The antisera was heat inactivated at $56^{\circ} \mathrm{C}$ for $30 \mathrm{~min}$. After inactivation the antisera was absorbed with $8 \times 10^{7}$ purified RH strain tachyzoites for $1.5 \mathrm{~h}$ at $37^{\circ} \mathrm{C}$. An antitachyzoite ELISA decreased from a preabsorption titer of 3,200 (IgG) and 1,600 (IgM) to a postabsorption titer of $<100$. Equal numbers of oocyst/sporozoite or tachyzoites were run on a $10 \%$ SDS-PAGE gel, transferred to nitrocellulose and incubated with tachyzoite absorbed antisera. Antisera-reactive antigens were identified with antihuman IgM antibody as before. Fig. 8 shows the marked reduction in antitachyzoite activity by the absorbed antisera. In comparison, several oocyst antigens remain in spite of absorption with tachyzoites. The most prominent of these oocyst/sporozoite antigens occur at $M_{\mathrm{r}}$ $<14,000,38,000,67,000$, and 190,000. A clear unstained

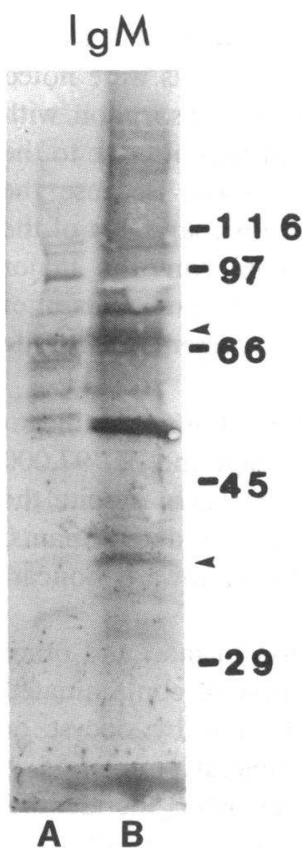

Figure 7. Nitrocellulose blot transfer of equal numbers of $(A) \mathrm{RH}$ strain tachyzoites and $(B)$ oocyst/sporozoites identified with the IgM fraction of convalescent-phase human antitoxoplasma antisera.

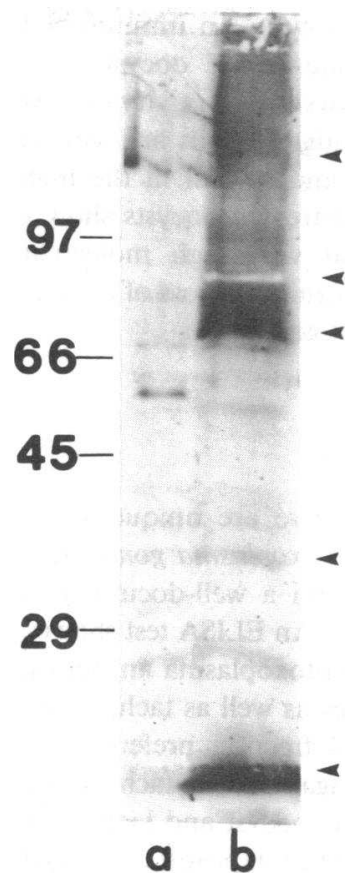

Figure 8. Nitrocellulose blot transfer of tachyzoite absorbed antitoxoplasma antisera. Equal numbers $\left(2 \times 10^{6}\right)$ of parasites were run on $10 \%$ polyacrylamide gels in the presence of SDS and electrophoretically transferred to nitrocellulose paper. Transferred strips were incubated with acute-phase antitoxoplasma antisera that had been absorbed with $8 \times 10^{7}$ tachyzoites. Antibody binding was assayed with peroxidase-labeled goat antihuman IgM. (a) RH strain tachyzoites and (b) oocyst/ sporozoites.

band persists at 70,000. Again, several faintly staining commonly shared oocyst/sporozoite and tachyzoite antigens that failed to be fully absorbed by the tachyzoites can be seen. Repeat absorption of the same antisera with $5.6 \times 10^{7}$ tachyzoites altered neither the results of the antitachyzoite ELISA nor identification of stage-specific oocyst/sporozoite antigens by this antisera.

Previous authors have indicated that treatment of tachyzoite antigens with $\mathrm{NaIO}_{4}$ reduced the reactivity of IgM and IgG antibodies in both an ELISA and Western blot analysis (7). The acute-phase IgM reacted most strongly to the oocyst antigens. We felt that it would be of interest to identify those oocyst antigens that were reduced by periodate treatment. To evaluate this, oocysts $\left(1 \times 10^{6}\right)$ were treated with $0.01 \mathrm{M}$ $\mathrm{NaIO}_{4}$ at $4^{\circ} \mathrm{C}$ for $18 \mathrm{~h}$, run on $10 \%$ SDS-PAGE gels, and stained with periodic acid. There was no difference between the treated and control preparation except for the loss of a

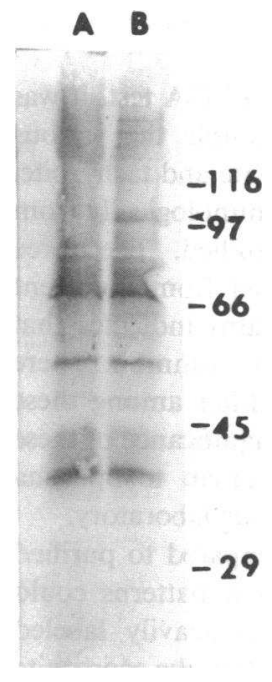

Figure 9. Nitrocellulose blot transfer of 2 $\times 10^{6} \mathrm{oocyst} / \mathrm{sporozoites}$ run on $10 \%$ polyacrylamide gels in the presence of SDS and transferred to nitrocellulose paper. Prior to SDS-PAGE, parasites were treated with a $0.01 \mathrm{M}$ solution of sodium periodate for 18 h at $4^{\circ} \mathrm{C}$. (A) Periodate-treated oocyst/sporozoites; $(B)$ untreated oocyst/sporozoites. Antibody binding of the IgM fraction of acutephase human antitoxoplasma antibody was determined with peroxidase-labeled goat antihuman IgM antibody. 
minor band of $M_{\mathrm{r}} 42,000$ (data not shown). An immunoblot analysis was done comparing periodate-treated oocysts to a control preparation. Fig. 9, lane $B$ shows the reactivity of IgM antibody to the untreated oocysts. Antigen bands are seen at $M_{\mathrm{r}} 38,000,67,000,97,000,190,000$, and several in the high molecular weight range. The periodate-treated oocysts show a definite reduction in the 97,000 and very high molecular weight bands. There appears to be no significant loss of activity in either the 67,000 or $190,000-M_{\mathrm{r}}$ antigen bands.

\section{Discussion}

The results presented establish that there are unique stagespecific oocyst/sporozoite antigens of Toxoplasma gondii that are identified by human antiserum from a well-documented outbreak of oocyst-transmitted infection. An ELISA test showed that acute and convalescent human antitoxoplasma antiserum reacted with purified oocyst/sporozoites as well as tachyzoites. Both the acute and convalescent IgG fraction preferentially expressed greater antibody activity against the tachyzoites. This difference in IgG titer between the oocyst and tachyzoite preparations is quite understandable. Host exposure to oocyst antigens is far less than tachyzoite antigens in both the amount and duration of antigens presented. Once the sporulated oocysts have entered the intermediate host's intestine they rapidly evolve within the epithelium to become invasive tachyzoites. Thus, there is only a short period of time during which an antibody response may be generated to these stage-specific antigens. We do not know the actual numbers of oocysts that were ingested by this group of patients although it was sufficient to elicit a stage-specific humoral response.

Absorption of the antisera with either oocyst/sporozoites or tachyzoites resulted in a reduction in the IgG and IgM antioocyst fractions. Oocyst/sporozoite absorption of the antisera had little effect on antitachyzoite activity. Both the IgG and IgM antioocyst titer fell after absorption with tachyzoites. This suggested that many of the oocyst antigens were crossreactive with tachyzoite antigens. The humoral response to systemic infection with toxoplasma tachyzoites would be much greater than the response to the briefly presented enteric oocyst/sporozoite. Therefore, one would expect lower antibody titers to these stage-specific oocyst antigens. In this setting, the ELISA test does not appear to be of adequate sensitivity to discern minor antigenic variations between these two parasite stages.

Because of the lack of sensitivity by the ELISA test, it was necessary to evaluate as completely as possible the various antigenic determinants on the oocyst/sporozoite and tachyzoite. These antigens differ biochemically and immunologically from the three tachyzoite strains that were studied. The three tachyzoite strains investigated were obtained from divergent sources. Western blot analysis of these strains indicates that the major tachyzoite antigens are shared in common. There are a variety of minor determinants that differ among these three tachyzoite strains. The immunologic significance of these strain variations and the correlation to human toxoplasma infection is presently being investigated in our laboratory.

When radiolabeled tachyzoites were compared to purified sporozoites, marked differences in iodination patterns could be appreciated. The predominance of two heavily labeled proteins of approximate $M_{\mathrm{r}} 25,000$ and 67,000 in the sporozoite preparation were noted. The silver stain also revealed a variety of minor weakly staining protein determinants that distinguish the oocyst from tachyzoite. Included in this group were several bands that were strongly labeled by radioiodination. The periodic acid stain of the oocysts confirmed a variety of unique carbohydrate containing stage-specific determinants that had not been identified by silver stain. The most prominent of these periodic acid-staining bands were the $M_{\mathrm{r}} 25,000$ and 67,000 bands observed with radioiodination and silver stain.

The immunologic significance of these various determinants was explored by Western blot analysis. In our previous study, we reported the inability to identify sporozoite/oocyst antigens by Western blot analysis by using radioiodinated antibody markers. Although the explanation for this failure remains unclear, there are several considerations. The absence of stagespecific antibody in the sample of human antitoxoplasma antisera tested or low stage-specific IgG titer (we assayed for IgG binding) are two possibilities. In spite of our previous failure using radioiodination techniques, both acute and convalescent antisera from a known oocyst-transmitted infection contained antibody that recognized several stage-specific sporozoite antigens. This specific antibody was present in the IgG and IgM fraction of these patients. The most prominent antigens were recognized by the acute-phase IgM fraction and are of approximate $M_{\mathrm{r}} 67,000$ and 190,000 . The IgG fraction of similarly pooled antisera identified bands of the same molecular weight plus a prominent $M_{\mathrm{r}} 25,000$ band. All of these bands have been observed by surface membrane radioiodination studies as well as silver and periodic acid staining of the oocyst/sporozoite. The acute-phase IgM fraction binds intensely to the $M_{\mathrm{r}} 67,000$ and 190,000 antigens. With convalescence the IgG fraction binds strongly to these same antigens. There is an appropriate shift in antibody class to these sporozoite antigens from acute IgM to convalescent IgG. Thus it does not appear that these antigens elicit a classspecific IgM reaction. A reduction in activity to these antigens is seen in the convalescent IgM fraction. A possible explanation for this may be the limited exposure to these antigens at the time of infection. In the absence of continued antigenic stimulation antibody titer rapidly declines. Absorption of the pooled antisera with tachyzoites failed to diminish the specific oocyst antibody response. Persistent oocyst bands were noted at $M_{\mathrm{r}} 25,000,38,000,67,000$, and 190,000 . Absorption with the two other tachyzoite strains was not carried out due to the limited supply of antisera available for study. Because the silver- and periodic acid-stained tachyzoite preparations of the three strains investigated appeared to share common major antigens, we do not feel that the residual oocyst bands after absorption with RH strain tachyzoites represent tachyzoite strain variation.

Periodate treatment of the oocysts resulted in no significant change in any major oocyst antigens except the $M_{\mathrm{r}} 97,000$ band which is a shared tachyzoite antigen. Thus, despite the strong periodic acid staining of these various determinants, most of the antibody appears to be directed toward noncarbohydrate determinants.

A major route of toxoplasma infection in man and other animals is believed to be via oral ingestion of contaminated material. The incidence of human infection by oocyst or bradyzoite-transmitted infection in the general population is unknown. We and others have recently described the identification of tissue cyst bradyzoite stage specific antigens $(22,23)$. 
The occurrence of stage-specific antibradyzoite antibody in humans is presently being investigated in our laboratory. The results of this present report suggest that humans infected by a presumed oocyst-acquired route develop antibody against unique stage-specific oocyst/sporozoite antigens. By using this data it will now be possible to screen a large number of infected patients with presumed oocyst-transmitted toxoplasmosis and determine the incidence of stage-specific antioocyst/ sporozoite antibody. The information obtained from that type of study would be beneficial in establishing the rate of oocystacquired toxoplasmosis in humans. This would be useful in providing guidelines for disease prevention in susceptible individuals.

\section{Acknowledgments}

This work was supported by grant AI-19613 from the National Institutes of Health, and by a basic research grant from the March of Dimes Birth Defects Foundation. Dr. Kasper is a recipient of Clinical Investigator Award 00509 from the National Institutes of Health.

\section{References}

1. Frenkel, J. K., J. P. Dubey, and N. L. Miller. 1970. Toxoplasma gondii in cats: fecal stages identified as coccidian oocysts. Science (Wash. DC). 167:893-896.

2. Hutchinson, W. M., J. F. Dunachie, J. Chr. Siim, and K. Work. 1970. Coccidian-like nature of Toxoplasma gondii. Br. Med. J. 1:142144.

3. Overdulve, J. P. 1970. The identity of Toxoplasma gondii. Proc. K. Ned. Akad. Wet. Ser. C Biol. Med. 73:129-141.

4. Sheffield, H. G., and M. L. Melton. 1970. Toxoplasma gondii: the oocyst, sporozoite, and infection of cultured cells. Science (Wash. DC). 167:892-893.

5. Kasper, L. H., M. S. Bradley, and E. R. Pfefferkorn. 1984. Identification of stage-specific sporozoite antigens of Toxoplasma gondii by monoclonal antibodies. J. Immunol. 132:443-449.

6. Kasper, L. H., J. H. Crabb, and E. R. Pfefferkorn. 1983. Purification of a major membrane protein of Toxoplasma gondii by immunoabsorption with a monoclonal antibody. J. Immunol. 130: 2407-2412.

7. Sharma, S. D., J. Mullenax, A. G. Araujo, H. A. Erlich, and J. S. Remington. 1983. Western blot analysis of the antigens of Toxoplasma gondii recognized by human IgM and IgG antibodies. $J$. Immunol. 131:977-983.

8. Handman, E., J. W. Goding, and J. S. Remington. 1980.
Detection and characterization of membrane antigens of Toxoplasma gondii. J. Immunol. 124:2578-2583.

9. Naot, Y., D. R. Guptill, J. Mullenax, and J. S. Remington. 1983. Characterization of Toxoplasma gondii antigens that react with human immunoglobulin $\mathbf{M}$ and immunoglobulin $\mathbf{G}$ antibodies. Infect. Immun. 41:331-338.

10. Ogata, K., T. Kasahara, K. Shioiri-Nakano, I. Igarashi, and M. Suzuki. 1984. Immunoenzymatic detection of three kinds of 43,000 molecular weight antigens by monoclonal antibodies in the insoluble fraction of Toxoplasma gondii. Infect. Immun. 43:1047-1053.

11. Hughes, H. P. A., F. Vanknapen, H. J. Atkinson, A. H. Balfour, and D. H. Lee. 1982. A new soluble antigen preparation of Toxoplasma gondii and its use in serological diagnosis. Clin. Exp. Immunol. 49:239-246.

12. Frenkel, J. K., and A. Ruiz. 1981. Endemicity of toxoplasmosis in Costa Rica. Am. J. Epidemiol. 113:254-269.

13. Ganley, J. P., and G. W. Comstock. 1980. Association of cats and toxoplasmosis. Am. J. Epidemiol. 111:238-246.

14. Teutsch, S. M., D. D. Juranek, A. Sulzer, J. P. Dubey, and R. K. Sikes. 1979. Epidemic toxoplasmosis associated with infected cats. N. Engl. J. Med. 300:695-699.

15. Beneson, M. W., E. T. Takafuji, S. M. Lemon, R. L. Greenup, and A. J. Sulzer. 1982. Oocyst transmitted toxoplasmosis associated with the ingestion of contaminated water. N. Engl. J. Med. 307:666669.

16. Pfefferkorn, E. R., and L. C. Pfefferkorn. 1976. Toxoplasma gondii: isolation and preliminary characterization of temperaturesensitive mutants. Exp. Parasitol. 39:365-373.

17. Kasper, L. H., J. H. Crabb, and E. R. Pfefferkorn. 1982 Isolation and characterization of a monoclonal antibody resistant antigenic mutant of Toxoplasma gondii. J. Immunol. 129:1694-1699.

18. Pfefferkorn, E. R., and L. H. Kasper. 1983. Toxoplasma gondii genetic crosses reveal phenotypic suppression of hydroxyurea resistance by fluorodeoxyuridine resistance. Exp. Parasitol. 55:207-218.

19. Wray, W., T. Boulikas, V. P. Wray, and R. Hancock. 1981. Silver staining of proteins in polyacrylamide gels. Anal. Biochem. 118: 197-203.

20. Dubray, G., and G. Bezard. 1982. A highly sensitive periodic acid-silver stain for 1,2-diol groups of glycoproteins and polysaccharides in polyacrylamide gels. Anal. Biochem. 119:325-329.

21. Towbin, H., T. Staehlin, and J. Gordon. 1979. Electrophoretic transfer of proteins from polyacrylamide gels to nitrocellulose sheets: procedure and some applications. Proc. Natl. Acad. Sci. USA. 76: 4350-4356.

22. Kasper, L. H., K. M. Currie, and M. S. Bradley. 1985. An unexpected response to vaccination with a purified major membrane tachyzoite antigen (P30) of Toxoplasma gondii. J. Immunol. In press.

23. Lunde, M. N., and L. Jacobs. 1983. Antigenic differences between endozoites and cystozoites of Toxoplasma gondii. J. Parasitol. 69:806-811. 